Pacific Journal of Mathematics

JORDAN TRIPLE SYSTEMS WITH COMPLETELY REDUCIBLE
DERIVATION OR STRUCTURE ALGEBRAS 


\title{
JORDAN TRIPLE SYSTEMS WITH COMPLETELY REDUCIBLE DERIVATION OR STRUCTURE ALGEBRAS
}

\section{ERHARD NEHER}

\begin{abstract}
We prove that a finite-dimensional Jordan triple system over a field $k$ of characteristic zero has a completely reducible structure algebra iff it is a direct sum of a trivial and a semisimple ideal. This theorem depends on a classification of Jordan triple systems with completely reducible derivation algebra in the case where $k$ is algebraically closed. As another application we characterize real Jordan triple systems with compact automorphism group.
\end{abstract}

The main topic of this paper is finite-dimensional Jordan triple systems over a field of characteristic zero which have a completely reducible derivation algebra.

The history of the subject begins with [7] where G. Hochschild proved, among other results, that for an associative algebra $\mathscr{Q}$ the derivation algebra is semisimple iff $\mathscr{Q}$ itself is semisimple. Later on $R$. D. Schafer considered in [18] the case of a Jordan algebra $\mathscr{q}$. His result was that Der $\mathscr{G}$ is semisimple if and only if $q$ is semisimple with each simple component of dimension not equal to 3 over its center. This theorem was extended by K.-H. Helwig, who proved in [6]:

Let $q$ be a Jordan algebra which is finite-dimensional over a field of characteristic zero. Then the following are equivlent:

(1) Der $\mathscr{g}$ is completely reducible and every derivation of $\mathscr{f}$ has trace zero,

(2) $g$ is semisimple,

(3) the bilinear form on Der $q$ given by $\left(D_{1}, D_{2}\right) \rightarrow \operatorname{trace}\left(D_{1} D_{2}\right)$ is non-degenerate and every derivation of $\mathcal{G}$ is inner.

After some preparations in $\S \S 1-3$ we will show in $\S 4$ that the same theorem holds for Jordan triple systems. The proof in this case is different from the Jordan algebra case. It relies on a classification of Jordan triple systems whose derivation algebras are completely reducible. It is easy to see that $V$ is an example for such a triple system, if

(a) $V$ is semisimple or if

(b) $V$ is trivial, i.e. all products vanish. 
Surprisingly there occurs another type of example:

(c) $V=X \oplus M$ with quadratic representation

$$
P(x \oplus m)(y \oplus n)=(2(x, y) x-(x, x) y) \oplus((x, x) n+2(x, y) m)
$$

where $(\cdot, \cdot)$ is a symmetric non-degenerate bilinear form on $X$. Our main result says that these are essentially all examples. To be more precise, let $V$ be a Jordan triple system over an algebraically closed field. Then the following are equivalent:

(I) Der $V$ is completely reducible.

(II) $V=V^{1} \oplus V^{2}$ is a direct sum of two ideals $V^{i}$ where-up to enumeration-one of the following two cases occurs:

( $\alpha) V^{1}$ is semisimple and $V^{2}$ is trivial,

( $\beta$ ) $V^{1}$ is semisimple and $V^{2}$ is a direct sum of ideals which are isomorphic to example (c).

In $§ 5$ we apply our results to characterize Jordan triple systems which have a compact automorphism group. Another application is given in $§ 6$ where we prove that a Jordan triple system $V$ has a completely reducible structure algebra iff $V$ is a direct sum of a semisimple and a trivial ideal.

The author thanks M. Koecher and K. McCrimmon for various useful suggestions and H. P. Petersson for having drawn his attention to [6].

\section{A review of known results.}

1.1. In this section we recall some known facts from the theory of Lie algebras and derive a few consequences. Throughout, $M$ denotes a finitedimensional vector space over a field $k$ of characteristic zero and $g$ is a subalgebra of $\mathfrak{g l}(M)$, the Lie algebra of all endomorphisms of $M$. By definition, $\mathfrak{g}$ is completely reducible (in $M$ ), if every $\mathfrak{g}$-invariant subspace has a $\mathfrak{g}$-invariant complement.

(1.1) ([2] §6.5, Théorème 4). $g$ is completely reducible iff $g$ is reductive and the center of $g$ consists of semisimple elements.

As a corollary we get

(1.2) If $\mathfrak{g}$ is completely reducible, then every ideal of $\mathfrak{g}$ is completely reducible, too.

(1.3) ([1] $§ 9.2$, Prop. 3). Let $K$ be an extension field of $k$. Then $g$ is completely reducible in $M$ iff $K \otimes \mathrm{g}$ is completely reducible in $K \otimes M$. 
(1.4) $g$ is called almost algebraic ("scindable" in [3]) if $g$ contains the semisimple and nilpotent components of all of its elements. The Lie algebra of an algebraic subgroup of $\operatorname{GL}(M)$ is almost algebraic ([5). In particular, the derivation algebra of an algebra or a triple system is almost algebraic (for a direct proof see [3] Chap. 7, §1, Prop. 4).

The following lemma is contained in [6] p. 28 for the case where $g$ is the derivation algebra of an algebra:

LEMMA 1.1. Let $\mathrm{g}$ be almost algebraic and assume that the bilinear form $\rho$ on $\mathrm{g}$ defined by $\rho(X, Y)=\operatorname{trace}(X Y)$ is non-degenerate. Then $\mathrm{g}$ is completely reducible.

Proof. By [2] §6.4, Prop. 5 we know that $g$ is reductive. Hence, using (1.1), the assertion follows, if we can show that the center $z$ of $g$ consists of semisimple elements. But $z$ is again almost algebraic ([3] §5.1, Corollaire 1 of Prop. 3) and thus it is enough to show that $z$ contains no nilpotent elements. Let $Z \in z$ be nilpotent, then $Z X$ is nilpotent for every $X \in \mathrm{g}$ and therefore $\operatorname{trace}(Z X)=0$, forcing $Z$ to be zero.

The following lemma is a trivial generalization of [2] $\$ 6.1$ Proposition 1:

Lemma 1.2. Let $\mathrm{g}$ be completely reducible. Define $\rho$ as in Lemma 1.1. Then $\rho$ is non-degenerate iff the restriction of $\rho$ to the center of $\mathrm{g}$ is non-degenerate.

Proof. By (1.1) $\mathfrak{g}$ is reducitve, i.e. $\mathfrak{g}=\mathfrak{g} \oplus[\mathfrak{g}, \mathfrak{g}]$, where $[\mathfrak{g}, \mathfrak{g}]$ is semisimple and $z$ is the center of $g$. Since $\rho$ is an invariant bilinear form, this decomposition is orthogonal relative to $\rho$. But the restriction of $\rho$ to $[\mathfrak{g}, \mathfrak{g}]$ is non-degenerate by [2] $\S 6.1$ Prop. 1 . Hence $\rho$ is nondegenerate iff the restriction of $\rho$ to $z$ is non-degenerate.

1.2. In this section we recall some well-known results about Jordan triple systems which are needed in the sequel. Thereby we also fix our notation.

A Jordan triple system as it is considered in this paper is a finite-dimensional vector space $V$ over a field $k$ of characteristic zero together with a trilinear map

$$
\{\cdots\}: V \times V \times V \rightarrow V:(u, v, w) \rightarrow\{u v w\}=: L(u, v) w
$$


which satisfies the following identities

$$
\begin{gathered}
\{u v w\}=\{w v u\} \\
{[L(u, v), L(x, y)]=L(\{u v x\}, y)-L(x,\{v u y\})}
\end{gathered}
$$

for all $u, v, w, x, y \in V$. In what follows we just speak of $V$ as the Jordan triple system without exhibiting the map $\{\cdots\}$.

The theory of Jordan triple systems is developed in [10], [11] and [14] for a more general class of triple systems. Since the proofs of our main results are only valid in the situation as defined above we restrict ourselves to this case from the very beginning.

For a Jordan triple system $V$ one defines the quadratic representation $P: V \rightarrow$ End $V$ by $P(x)=\frac{1}{2}\{x y x\}$. This is in fact a quadratic map since its linearization $P(x, z)=P(x+z)-\{(x)-P(z)$ satisfies $P(x, z) y=$ $\{x y z\}$. As a result, $V$ is uniquely determined by $P$.

There are many identitites valid in $V$, see e.g. [11] $\S 2$. We need the following which is a translation of (JP12) in [11]:

$$
L(x, y) P(z)+P(z) L(y, x)=P(z,\{x y z\}) .
$$

There are several Lie algebras associated with Jordan triple systems. In this paper we are concerned with the structure algebra and the derivation algebra of $V$. The structure algebra appears in connection with the derivation algebra of the Jordan pair $(V, V)$ associated to $V$ : By definition, $(A, B) \in \operatorname{Der}(V, V)$ if

$$
[A, L(x, y)]=L(A x, y)+L(x, B y)
$$

and

$$
[B, L(x, y)]=L(B x, y)+L(x, A y)
$$

holds for all $x, y \in V$. Der $(V, V)$ is a subalgebra of $(\mathfrak{g l}(V), \mathfrak{g l}(V))$ with respect to componentwise multiplication. The structure algebra $\mathcal{T}(V)$ of $V$ is the image of $\operatorname{Der}(V, V)$ under the projection map onto the first component:

$$
\operatorname{Der}(V, V) \rightarrow \mathcal{T}(V):(A, B) \rightarrow A .
$$

We point out that this map is in general not injective. It is an immediate consequence of (1.6) that

$$
(L(u, v),-L(v, u)) \in \operatorname{Der}(V, V)
$$

for all $u, v \in V$, correspondingly $L(u, v) \in \mathcal{T}(V)$. The subspace of $\mathcal{T}(V)$ spanned by $\{L(u, v) ; u, v \in V\}$ is an ideal of $\mathscr{T}(V)$ called the inner structure algebra and denoted by inn $\mathcal{T}(V)$. 
A derivation of $V$ is a $k$-linear map $D: V \rightarrow V$ satisfying $D\{x y z\}=$ $\{D z, y, z\}+\{x, D y, z\}+\{x, y, D z\}$ for all $x, y, z \in V$, i.e. an endomorphism $D$ with $(D, D) \in \operatorname{Der}(V, V)$. The space Der $V$ of all derivations of $V$ is a Lie subalgebra of $\mathscr{T}(V)$. Since $(L(u, v),-L(v, u)) \in \operatorname{Der}(V, V)$ it follows easily that

$$
\Delta(u, v):=L(u, v)-L(v, u) \in \operatorname{Der} V .
$$

The span of $\{\Delta(u, v) ; u, v \in V\}$ is an ideal of Der $V$ denoted by inn Der $V$. The elements of inn Der $V$ are called inner derivations.

If $K$ is an extension field of $k$ and $V$ a Jordan triple system over $k$, the triple product of $V$ can be naturally extended to $K \otimes V$. In this way $K \otimes V$ becomes a Jordan triple system. As for algebras one proves

$$
\mathscr{T}(K \otimes V)=K \otimes \mathcal{T}(V), \quad \operatorname{Der}(K \otimes V)=K \otimes \operatorname{Der} V .
$$

A subsystem of $V$ resp. an ideal of $V$ is a subspace $U$ of $V$ such that $\{U U U\} \subset U$ resp. $\{V V U\}+\{V U V\} \subset U$. One calls $V$ simple if $\{V V V\}$ $\neq 0$ and $V$ contains only the trivial ideals 0 and $V$. Since $\{V V V\}$ is an ideal, we have in this case $\{V V V\}=V$.

The $k$-linear span of all products in $V$ of degree $>n$ is denoted by $V_{(n)}$, where $\{x y z\}$ resp. $\{x y\{u v w\}\}$ or $\{x\{y u v\} w\} \ldots$ is considered to be of degree 3 resp. $5 \ldots$ Obviously, $V=V_{(1)} \supset V_{(3)} \supset V_{(5)} \supset \cdots$ and each $V_{(n)}$ is an ideal of $V$. If $V_{(n)}=0$ for some $n$, then $V$ is said to be nilpotent.

In the situation considered here every Jordan triple system $V$ contains a unique maximal nilpotent ideal $\operatorname{Rad} V$ which is called the radical of $V$. Since $(\operatorname{Rad} V, \operatorname{Rad} V)$ is the radical of the Jordan pair $(V, V)$, it is invariant under the automorphism group of $(V, V)$ and thus also under its Lie algebra (see e.g. [5] II §8 Prop. 6) which is $\operatorname{Der}(V, V)$. Hence

$$
\operatorname{Rad} V \text { is invariant under } \mathcal{T}(V) \text {. }
$$

We will need another property of $\operatorname{Rad} V$ which follows from [11] Theorem 14.10:

$$
\text { The subspace } L(V, \operatorname{Rad} V)+L(\operatorname{Rad} V, V) \text { of End } V
$$
consists of nilpotent endomorphisms.

A Jordan triple $V$ is called semisimple, if its radical vanishes. It is known ([14] §11) that the following properties are equivalent: the trace form $\sigma$ of $V$ defined by $\sigma(u, v)$ $=\frac{1}{2} \operatorname{trace}(L(u, v)+L(v, u))$ is non-degenerate,

(1.11.c) $K \otimes V$ is semisimple for every extension field $K$ of $k$, 
It is also proved in [14] $\$ 11.6$ :

If $V$ is semisimple, then $\mathscr{T}(V)=\operatorname{inn} \mathscr{T}(V)$ and Der $V=$

(1.12) inn Der $V$. Moreover, the projection $\operatorname{Der}(V, V) \rightarrow \mathscr{T}(V)$ is an isomorphism.

The existence of a Wedderburn decomposition of $V$ was show in [9]:

For every Jordan triple system $V$ (as defined above)

(1.13) there exists a semisimple subsystem $S$ of $V$ such that $V=\operatorname{Rad} V \oplus S$.

It follows from [14] 11.3 Theorem 4:

If $V$ is a simple Jordan triple system, then either $\sigma(V)$ operates irreducibly on $V$ or $V=V^{+} \oplus V^{-}$is the direct sum of two $\mathcal{T}(V)$-irreducible subspaces $V^{+}$and $V^{-}$ satisfying

$$
\left\{V^{\varepsilon} V^{-\varepsilon} V^{\varepsilon}\right\} \subset V^{\varepsilon}, \quad\left\{V^{\varepsilon} V^{\varepsilon} V\right\}=0 \quad \text { for } \varepsilon= \pm .
$$

Using (1.11) we get as an easy corollary:

(1.15) If $V$ is semisimple, then $\sigma(V)$ is completely reducible.

LEMMA 1.3. Let $V$ be semisimple and define the bilinear form $\rho$ on $\mathcal{T}(V)$ by $\rho(X, Y)=\operatorname{trace}(X Y)$. Then $\rho$ is non-degenerate.

Proof. First we make two reductions:

(1) Let $K$ be an extension field of $k$. Then $K \otimes V$ is a semisimple Jordan triple system by (1.11.c), $D \otimes \mathcal{T}(V)=\mathcal{T}(V \otimes K)$ and $\rho$ defined for $K \otimes V$ is the bilinear extension of $\rho \mid \sigma(V)$. Without loss of generality we may therefore only consider the case where $k$ is algebraically closed.

(2) If $V=V^{1} \oplus \cdots \oplus V^{n}$ is the decomposition of $V$ as sum of its simple ideals, then we have the corresponding decomposition $\sigma(V)=$ $\mathcal{T}\left(V^{1}\right) \oplus \cdots \oplus \mathcal{T}\left(V^{n}\right)$ where $\mathcal{T}\left(V^{i}\right)$ is canonically imbedded in $\mathscr{T}(V)$. Since this decomposition is orthogonal relative to $\rho$ it is enough to consider the case where $V$ is simple. Because $\mathscr{T}(V)$ is completely reducible we can apply Lemma 1.2 and see that we have to show that the restriction of $\rho$ to the center $z$ of $\mathcal{T}(V)$ is non-degenerate.

If $\mathcal{T}(V)$ is irreducible, then the centralizer of $\mathscr{T}(V)$ in $\operatorname{End}(V)$ equals $k$ Id and contains $z$. But $\mathrm{Id} \in \mathcal{T}(V)$ since $(\mathrm{Id},-\mathrm{Id}) \in \operatorname{Der}(V, V)$ and thus $z=k$ Id. Obviously, $\rho \mid z$ is nondegenerate. 
If $\mathcal{T}(V)$ is not irreducible, then, by (1.14), we know that $V=V^{+} \oplus V^{-}$ is a direct sum of $\mathcal{T}(V)$-irreducible subspaces $V^{+}$and $V^{-}$. Again, we have Id $\in \mathcal{T}(V)$, i.e.

$$
\mathrm{Id}=\sum_{i} L\left(u_{i}, v_{i}\right)=\sum_{i} L\left(u_{i}^{+}, v_{i}^{-}\right)+\sum_{i} L\left(u_{i}^{-}, v_{i}^{+}\right),
$$

thus

$$
\operatorname{Id}\left|V^{+}=\sum_{i} L\left(u_{i}^{+}, v_{i}^{-}\right) \in \mathcal{T}(V), \quad \operatorname{Id}\right| V^{-}=\sum_{i} L\left(u_{i}^{-}, v_{i}^{+}\right) \in \mathcal{T}(V) .
$$

Every endomorphism of $V$ which commutes with $\operatorname{Id} \mid V^{\varepsilon}, \varepsilon= \pm$, leaves invariant $V^{\varepsilon}$. This implies $z(\sigma(V))=k \mathrm{Id}\left|V^{+} \oplus k \mathrm{Id}\right| V^{-}$. Obviously, $\rho \mid z(\mathcal{T}(V))$ is non-degenerate.

COROllaRY 1.4. Let $V$ be semisimple. Then Der $V$ is completely reducible and the bilinear form $\rho$ on Der $V$ defined by $\rho(X, Y)=\operatorname{trace}(X Y)$ is non-degenerate.

Proof. Since Der $V$ is almost algebraic it is, by Lemma 1.1, enough to show the second assertion. Let $T^{*}$ denote the adjoint of $T \in$ End $V$ relative to the trace form $\sigma$ of $V$. Taking traces in the defining identitites for $(A, B) \in \operatorname{Der}(V, V)$ shows $B=-A^{*}$. Because also $(B, A) \in$ $\operatorname{Der}(V, V)$ we see that $\mathscr{T}(V)$ is invariant under $*$ and thus $\mathscr{T}(V)=\mathcal{T}_{+}(V)$ $\oplus \mathcal{T}_{-}(V)$ where $\mathcal{T}_{ \pm}(V)=\left\{T \in \mathcal{T}(V) ; T^{*}= \pm T\right\}$. since $D \in \operatorname{Der} V$ iff $(D, D) \in \operatorname{Der}(V, V)$ we obtain $\operatorname{Der} V=\mathcal{T}(V)$. Let $S \in \mathcal{T}_{+}(V)$ and $T \in$ $\sigma_{-}(V)$. Then

$$
\operatorname{trace}(S T)=\operatorname{trace}(S T)^{*}=\operatorname{trace}\left(T^{*} S^{*}\right)=-\operatorname{trace}(T S)=-\operatorname{trace}(S T)
$$

shows that $\mathcal{T}_{+}(V)$ and $\mathcal{T}_{-}(V)$ are orthogonal relative to $\rho$, in particular $\rho \mid$ Der $V$ is non-degenerate.

An element $e \in V$ such that $P(e) e=e$ is called a tripotent. Every tripotent of $V$ induces a Peirce decomposition

$$
V=V_{2}(e) \oplus V_{1}(e) \oplus V_{0}(e)
$$

where $V_{i}(e)=\{x \in V ;\{e e x\}=i x\}$ for $i=0,1,2$. The Peirce spaces have the following multiplication rules:

$\left\{V_{i}(e) V_{j}(e) V_{k}(e)\right\} \subset V_{i-j+k}(e)$, in particular, $V_{i}(e)$ is a subsystem of $V$.

$V_{2}(e)=\operatorname{im} P(e)$ and $P(e) \mid V_{2}(e)$ is an involutorical automorphism of $V_{2}(e)$, hence 
(1.18) $=\varepsilon x\}$ for $\varepsilon= \pm$, where we are using the abbreviation $P(e) x=\bar{x}$.

Together with the algebra product $x y=\frac{1}{2}\{x e y\}$ the subspace $V_{2}(e)$ becomes a Jordan algebra denoted by $V_{2}(e)^{(e)}$. The triple product of $V_{2}(e)$ can be expressed by the algebra product

$$
\{x y z\}=2[x(\bar{y} z)-\bar{y}(x z)+(x \bar{y}) z] \text { for } x, y, z \in V_{2}(e) .
$$

Two nonzero tripotents $e$ and $f$ are called orthogonal if $f \in V_{0}(e)$ which is equivalent to $e \in V_{0}(f)$. A nonzero tripotent $e$ is called minimal, if $e$ cannot be written as the sum of two orthogonal tripotents. We remark that in this case $e$ is a primitive idempotent of the Jordan algebra $\left[V_{2}^{+}(e)\right]^{(e)}$. Using well-known results for Jordan algebras (see e.g. [4] IV §5.5) we get

$$
\text { If } k \text { is algebraically closed and } e \in V \text { is a minimal }
$$

$$
\text { tripotent, then } V_{2}^{+}(e)=k e \oplus \operatorname{Rad} V_{2}^{+}(e) \text {. }
$$

An orthogonal system is a tuple $\left(e_{1}, \ldots, e_{r}\right)$ of pairwise orthogonal tripotents. In this case $V$ has a Peirce decomposition relative to $\left(e_{1}, \ldots, e_{r}\right)$, i.e. a decomposition

$$
\begin{aligned}
V & =\bigoplus_{0 \leq i \leq j \leq r} V_{i j} \text { where } \\
V_{00} & =\bigcap_{i=1}^{r} V_{0}\left(e_{i}\right), \quad V_{i i}=V_{2}\left(e_{i}\right), \quad 1 \leq i \leq r, \\
V_{i j} & =V_{j i}=V_{1}\left(e_{i}\right) \cap V_{1}\left(e_{j}\right) \quad \text { for } 1 \leq i<j \leq r, \\
V_{0 j} & =V_{j 0}=V_{1}\left(e_{j}\right) \cap \bigcap_{i \neq j} V_{0}\left(e_{i}\right) \quad \text { for } 0<j \leq r .
\end{aligned}
$$

We also put for $1 \leq i \leq r$

$$
V_{i i}=V_{i i}^{+} \oplus V_{i i}^{-} \quad \text { with } V_{i i}^{\varepsilon}=V_{2}^{\varepsilon}\left(e_{i}\right) .
$$

The Peirce spaces satisfy the following multiplication rules

$$
\left\{V_{j k} V_{k l} V_{l m}\right\} \subset V_{j n} \text {, and all other types of products are }
$$
zero.

The radical of $V$ splits relative to $\left(e_{1}, \ldots, e_{r}\right)$. In particular, one derives from [17] Lemma 6:

$$
\text { If } V \text { is semisimple, then all the Peirce spaces } V_{\mathrm{ij}} \text { are }
$$
semisimple, too. 
In general, $V$ need not contain any nonzero tripotents. However, one knowns ([10] 3.3):

If $k$ is algebraically closed and $V$ is semisimple, then $V$ contains nonzero tripotents.

By induction, we get:

Let $k$ be algebraically closed and $V$ semisimple. Then $V$ (1.26) contains a maximal set of orthogonal minimal tripotents. The corresponding Peirce space $V_{00}$ is zero.

2. Examples of Jordan triple systems with completely reducible derivation algebra.

2.1. As in the preceding section let $k$ be a field of characteristic 0 . Assume $X$ is a finite-dimensional vector space over $k$ and $(\cdot, \cdot): X \rightarrow k$ is a symmetric bilinear form. We put

$$
\{x y z\}=2[(x, y) z+(z, y) x-(x, z) y] .
$$

It is well-known ([10]), that this triple system is in fact a Jordan triple system, called the Jordan triple (system) of the quadratic form $(\cdot, \cdot)$ and denoted by $[X ;(\cdot, \cdot)]$. Obviously, its quadratic representation is given by

$$
P(x)=2(x, y) x-(x, x) y .
$$

From (2.1) one easily derives that the trace form $\sigma$ is given by $\sigma(x, y)=$ $2(x, y) \operatorname{dim} X$. Hence, by (1.11.b), we get

A Jordan triple system of a quadratic form is semisimple iff the form is non-degenerate.

Also, the next assertion trivially follows from (2.2) and (2.1):

A nonzero element $e$ of $X$ is a tripotent iff $(e, e)=1$. In

(2.4) this case $X=X_{2}(e)=k e \oplus X_{2}^{-}(e)$ with $X_{2}^{-}(e)=\{x$ $\in X ;(e, x)=0\}$.

Jordan triples of quadratic forms occur in the following connection:

LEMMA 2.1. Let $V$ be a Jordan triple and $e \in V$ a tripotent with $V=V_{2}(e)$ and $V_{2}^{+}(e)=k e$. Then there exists a unique quadratic form $(\cdot, \cdot)$ on $V$ such that $V$ is the Jordan triple of $(\cdot, \cdot)$.

The form is given by

$$
(\alpha e+x, \beta e+y)=\alpha \beta-\frac{1}{2}\{x e y\}, \quad x, y \in V_{2}^{-}(e) .
$$


Proof. Since $P(e)$ is an involutorical automorphism of $V$ we get $\{x e y\} \in V_{2}^{+}(e)=k e$ for every $x, y \in V_{2}^{-}(e)$. This shows that our form is - up to a trivial identification-well-defined. From (1.19) we derive $P(u) v=2 u(u \bar{v})-u^{2} \bar{v}, u v=\frac{1}{2}\{u e v\}$. Since

$$
u v=\left(\alpha e+u^{-}\right)\left(\beta e+v^{-}\right)=\left(\alpha \beta-\left(u^{-}, v^{-}\right)\right) e+\alpha v^{-}+\beta u^{-}
$$

it is easily seen that $(2.2)$ is valid in $V$, i.e. $V$ is the Jordan triple of $(\cdot, \cdot)$. The uniqueness follows form (2.4) and (2.1).

There is a general method for constructing new Jordan triple systems out of old ones which is stated in the following

THEOREM 2.2 ([14]10 Theorem 2). Let $V$ be a Jordan triple system with quadratic representation $P$ and $\varphi \in$ End $V$ such that $P(\varphi x)=\varphi P(x) \varphi$ for all $x \in V$. Then the quadratic map $P_{(\varphi)}$ where $P_{(\varphi)}(x)=P(x) \varphi$ defines on $V$ the structure of a Jordan triple system.

We use this theorem in the following special case:

LEMMA 2.3. Let $X, M$ be nonzero finite dimensional vector spaces over $k$ and $(\cdot, \cdot)$ a symmetric non-degenerate bilinear form on $X$. We define a quadratic representation on $V=X \oplus M$ by

$$
\begin{aligned}
P(x \oplus m)(y \oplus n)= & (2(x, y) x-(x, x) y) \\
& \oplus((x, x) n+2(x, y) m)
\end{aligned}
$$

where $x, y \in X$ and $m, n \in M$. Then

(a) $(V, P)$ is a Jordan triple system,

(b) the decomposition $V=X \oplus M$ is a Wedderburn decomposition with the semisimple part $X$ and $M=\operatorname{Rad} V$,

(c) $\{V V V\}=V$.

(d) the derivation algebra of $V$ consists of the mappings $D(x \oplus m)=$ $D_{1} x \oplus D_{2} m$ where $D_{1} \in \operatorname{Der}[X ;(\cdot, \cdot)]$ and $D_{2} \in \mathfrak{g} \mathfrak{l}(M)$ is arbitrary. In particular, Der $V$ is completely reducible.

(e) $\mathcal{T}(V)$ and inn $\mathcal{T}(V)$ are not completely reducible.

Proof. (a) We extend $(\cdot, \cdot)$ to a bilinear form on $V$ by setting $(x \oplus m, y \oplus n)=(x, y)$ and define $\varphi \in$ End $V, \varphi(x \oplus m)=x-m$. Let $Q$ denote the quadratic representation of the Jordan triple system $[V ;(\cdot, \cdot)]$. 
Then, using (2.2), we derive

$$
\begin{aligned}
& Q(\varphi(x+m))(y+n)=Q(x-m)(y+n) \\
& \quad=2(x, y)(x-m)-(x, x)(y+n) \\
& \quad=\varphi[2(x, y)(x+m)-(x, x)(y-n)]=\varphi Q(x+m) \varphi(y+n) .
\end{aligned}
$$

Thus, by Theorem 2.2, we conclude that $P=Q_{(\varphi)}$ defines on $V$ a Jordan triple system. But

$$
\begin{aligned}
P(x+m)(y+n) & =Q(x+m)(y-n) \\
& =2(x, y)(x+m)-(x, x)(y-n)
\end{aligned}
$$

which is just (2.5).

(b) From (2.5) and (2.2) it follows that the subsystem $X$ of $V$ is the Jordan triple system of the quadratic form $(\cdot, \cdot) \mid X$. Hence, by $(2.3)$, it is semisimple. On the other hand, $\{V V M\}=M=\{V M V\}$ and $\{M M M\}=$ 0 shows that $M$ is a nilpotent ideal of $V$ and therefore $M \subset \operatorname{Rad} V$. Now (b) easily follows.

(c) Since $X$ is semisimple we have $\{X X X\}=X$. Moreover, there exists $x \in X$ with $(x, x) \neq 0$. Then $P(x) M=M$. Altogether, $\{V V V\}=V$ follows.

(d) Let $D$ be a derivation, $x \in X$. So $D x=y \oplus m$ for some $y \in X$ and $m \in M$. Because $D P(x) x=P(x) D x+\{x x D x\}$ we derive from (2.5)

$$
(x, x)(y+m)=P(x) y+\{x x y\}+(x, x) m+2(x, x) m,
$$

hence $(x, x) m=0$. But since the $x$ with $(x, x) \neq 0$ form an algebraically dense subset of $X$ we have $D X \subset X$. By (1.9) we also know $D M \subset M=$ $\operatorname{Rad} V$. A straightforward computation using $\left(D_{1} x, x\right)=0$ for every $D_{1} \in$ $\operatorname{Der}[X ;(\cdot, \cdot)]$ and $x \in X$ shows now that $D \in \operatorname{Der} V$ iff $D \mid X \in$ $\operatorname{Der}[X ;(\cdot, \cdot)]$ and $D \mid M \in \mathfrak{g} l(M)$. Since $\operatorname{Der}[X ;(\cdot, \cdot)]$ is completely reducible in $X$ by Corollary 1.4 it follows that Der $V$ is completely reducible in $V$.

(e) Since inn $\mathcal{T}(V)$ is an ideal of $\mathscr{T}(V)$ it follows from (1.2) that is enough to show: inn $\mathscr{T}(V)$ is not completely reducible. Putting $\left(x y^{*}\right)(z):=(y, z) x$ we get

$$
\begin{aligned}
& \frac{1}{2} L(x \oplus m, y \oplus n) \\
& \quad=\left((x, y) \operatorname{Id}_{X}+x y^{*}-y x^{*}\right) \oplus\left(n x^{*}+m y^{*}+(x, y) \operatorname{Id}_{M}\right) .
\end{aligned}
$$

This easily implies $\operatorname{Id}_{V}, x y^{*}-y x^{*}$ and $n x^{*} \in \operatorname{inn} \mathcal{T}(V)$ for all $x, y \in X$, $n \in M$. Since the skew adjoint endomorphisms of $X$ are spanned by $x y^{*}-y x^{*}, x, y \in X$, and $\operatorname{Hom}(X, M)$ is spanned by $n x^{*}, n \in M, x \in X$ 
we get

$$
\text { inn } \mathcal{T}(V)=k \operatorname{Id} \oplus \operatorname{so}(X) \oplus \operatorname{Hom}(X, M)
$$

as direct sum of vector spaces. It follows that $0 \neq \operatorname{Hom}(X, M) \subset$ inn $\mathcal{T}(V)$ is an abelian ideal. If inn $\mathcal{T}(V)$ is completely reducible, then $\operatorname{Hom}(X, M)$ is also completely reducible and (1.1) implies that every element of $\operatorname{Hom}(X, M)$ is semisimple, but $\operatorname{Hom}(X, M)$ consists of nilpotent endomorphisms.

REMARK 2.4. (a) We will see later (Theorem 2.7) that there are other types of Jordan triple systems whose derivation algebras are completely reducible. In the sequel we will refer to a Jordan triple system considered in Lemma 2.3 as Example 2.3.

(b) For an Example 2.3 with $V=X \oplus M$ it is easy to see that $M$ is a bimodule for $X$ and $V$ is the split-null-extension of $X$ by $M$.

(c) One can show $\mathcal{T}(V)=k \operatorname{Id} \oplus \operatorname{so}(X(\cdot, \cdot)) \oplus \operatorname{Hom}(V, M)$, but we don't need this in the sequel.

2.2. We consider the situation where the Jordan triple system $V$ is a direct sum of two ideals $V^{1}$ and $V^{2}$. We put

$$
\begin{aligned}
& \mathfrak{g l}_{0}(V):=\left\{A \in \mathfrak{g} \mathfrak{l}(V) ; A V^{i} \subset V^{i}, i=1,2\right\}, \\
& \mathfrak{g l}_{1}(V):=\left\{A \in \mathfrak{g} \mathfrak{l}(V), A V^{1} \subset V^{2}, A V^{2} \subset V^{1}\right\}
\end{aligned}
$$

Then $\mathfrak{g} \mathfrak{l}(V)=\mathfrak{g l}_{0}(V) \oplus \mathfrak{g l}_{1}(V)$ is a $\mathbf{Z}_{2}$-grading of the Lie algebra $\mathfrak{g} \mathfrak{l}(V)$. Correspondingly,

$$
(\mathfrak{g} \mathfrak{l}(V), \mathfrak{g} \mathfrak{l}(V))=\mathfrak{g} \mathfrak{l}_{0}(V, V) \oplus \mathfrak{g} \mathfrak{l}_{1}(V, V)
$$

where $\mathfrak{g l}_{i}(V, V)=\left(\mathfrak{g l}_{i}(V), \mathfrak{g l}_{i}(V)\right)$.

Lemma 2.5. Let $V=V^{1} \oplus V^{2}$ be a direct sum of two ideals $V^{i}$. Then

(a) $\operatorname{Der}(V, V)=\operatorname{Der}_{0}(V, V) \oplus \operatorname{Der}_{1}(V, V)$ where $\operatorname{Der}_{i}(V, V)=$ $\operatorname{Der}(V, V) \cap \mathfrak{g l}_{i}(V, V)$

(b) $\operatorname{Der}_{0}(V, V)=\operatorname{Der}\left(V^{1}, V^{1}\right) \oplus \operatorname{Der}\left(V^{2}, V^{2}\right)$ is a direct sum of the ideals $\operatorname{Der}\left(V^{i}, V^{i}\right) \subset \operatorname{Der}_{0}(V, V)$.

(c) if $(A, B) \in \mathfrak{g l}_{1}(V, V),(A, B) \in \operatorname{Der}_{1}(V, V)$ iff

$$
\begin{aligned}
& A\left\{V^{i} V^{i} V^{i}\right\}=\left\{A V^{i}, V^{j}, V^{j}\right\}=\left\{V^{j}, A V^{i}, V^{j}\right\}=0 \quad \text { and } \\
& B\left\{V^{i} V^{i} V^{i}\right\}=\left\{B V^{i}, V^{j}, V^{j}\right\}=\left\{V^{j}, B V^{j}, V^{j}\right\}=0
\end{aligned}
$$

for $(i, j)=(1,2),(2,1)$. 
Proof (a) Since $\pi: x^{1} \oplus x^{2} \rightarrow x^{1} \oplus-x^{2}$ defines an automorphism of $V$ we have $(\pi, \pi) \in \operatorname{Aut}(V, V)$, thus

$$
(A, B) \rightarrow(\pi A \pi, \pi B \pi)
$$

is an involutive automorphism of $\operatorname{Der}(V, V)$ and the decomposition in the assertion is just the eigenspace decomposition of this automorphism.

(b) is obvious and (c) is a straightforward verification.

We will need the following corollary later:

Corollary 2.6. Let $V=V^{1} \oplus V^{2}$ be a direct sum of two ideals $V^{i}$, $i=1,2$.

(a) $\operatorname{Der} V=\operatorname{Der}_{0} V \oplus \operatorname{Der}_{1} V$ where $\operatorname{Der}_{i} V=\operatorname{Der} V \cap \mathfrak{g l}_{i}(V)$

(b) $\operatorname{Der}_{0} V=\operatorname{Der} V^{1} \oplus \operatorname{Der} V^{2}$ is a direct sum of the two ideals Der $V^{i}$ $\subset \operatorname{Der}_{0} V$.

(c) Let $D \in \mathfrak{g l}_{1}(V)$. Then $D \in \operatorname{Der}_{1} V$ iff

$$
D\left\{V^{i} V^{i} V^{i}\right\}=\left\{D V^{i}, V^{j}, V^{j}\right\}=\left\{V^{j}, D V^{i}, V^{j}\right\}=0
$$

for $(i, j)=(1,2),(2,1)$.

Proof. This is an immediate consequence of Lemma 2.5, if one notes that the automorphism $(A, B) \rightarrow(\pi A \pi, \pi B \pi)$ leaves $\{(D, D) \in$ $\operatorname{Der}(V, V)\} \sim \operatorname{Der} V$ invariant.

THEOREM 2.7. Let $V=V^{1} \oplus V^{2}$ be a direct sum of two ideals $V^{i}$.

(a) If $V^{1}$ is semisimple and $V^{2}$ is trivial, i.e. $\left\{V^{2} V^{2} V^{2}\right\}=0$, then $\sigma(V)$ and $\operatorname{Der}(V)$ are completely reducible.

(b) If $V^{1}$ is semisimple and $V^{2}$ is nonzero and a direct sum of ideals each of which is isomorphic to an Example 2.3, then Der $V$ is completely reducible, but $\mathcal{T}(V)$ is not.

Proof. (a) Since $V_{(3)}^{1}=V^{1}$ by (1.11.d) and since $V^{1}$ contains no subspace $I$ with $\left\{I V^{1} V^{1}\right\}=0=\left\{V^{1} I V\right\}$, it follows from Lemma 2.5 that $\operatorname{Der}_{1}(V, V)=0$. Consequently $\mathscr{T}(V)=\mathscr{T}\left(V^{1}\right) \oplus \mathcal{T}\left(V^{2}\right)$ is a direct sum of ideals $\mathscr{T}\left(V^{i}\right) \subset \mathcal{T}(V)$. But $\mathscr{T}\left(V^{1}\right)$ is completely reducible by (1.15) and $\mathscr{T}\left(V^{2}\right)=\mathfrak{g} \mathfrak{l}\left(V^{2}\right)$ is also completely reducible, which implies that $\mathscr{T}(V)$ is completely reducible. Since Der $V$ is a subalgebra of $\mathscr{T}(V)$ we have Der $V=\operatorname{Der} V^{1} \oplus \operatorname{Der} V^{2}$ with $\operatorname{Der} V^{2}=\mathrm{gl}\left(V^{2}\right)$. Thus the remaining assertion is a consequence of Corollary 1.4. 
(b) Here $V$ is a direct sum of ideals $I^{i}$ which are semisimple or isomorphic to an Example 2.3. By (1.11.d) and Lemma 2.3.c we know $I_{(3)}^{i}=I^{i}$ for all ideals $I^{i}$. Thus, by induction $\operatorname{Der}_{1}(V, V)=0$ and $\mathscr{T}(V)=$ $\bigoplus \mathcal{T}\left(I^{i}\right)$, Der $V=\bigoplus_{i} \operatorname{Der} I^{i}$.

Since at least one $I^{i}$ is an Example 2.3, the ideal $\mathscr{T}\left(I^{i}\right)$ of $\mathcal{T}(V)$ is not completely reducible (Lemma 2.3.e) and hence $\sigma(V)$ is not completely reducible by (1.2). However, all ideals Der $I^{i}$ are completely reducible and therefore Der $V$ is completely reducible.

3. The center of a Jordan triple system. The center of a Jordan triple system $V$ is defined as the subspace

$$
C(V)=\{c \in V ; L(c, v)=L(v, c) \text { for all } v \in V\} .
$$

LEMMA 3.1. (a) For $u, v \in V$ and $c, c_{1}, c_{2} \in C(V)$ we have

$$
\begin{gathered}
\{c v u\}=\{v c u\}=\{c u v\}, \\
{\left[L(u, v), L\left(c_{1}, c_{2}\right)\right]=0=\left[P(u, v), L\left(c_{1}, c_{2}\right)\right] .}
\end{gathered}
$$

(b) $C(V)$ is a subsystem of $V$.

Proof. (a) Every $c \in C(V)$ satisfies $\{c v u\}=\{v c u\}$ for all $u, v \in V$. Since the right side of this equation is symmetric in $u$ and $v$ we also get $\{c v u\}=\{c u v\}$ for all $u, v \in V$. From (1.6) we derive for $c \in C(V)$ : $[L(u, v), L(c, c)]=L(\{u v c\}, c)-L(c,\{v u c\})=0$ because $\{u v c\}=$ $\{v u c\}$. Now $\left[L(u, v), L\left(c_{1}, c_{2}\right)\right]=0$ follows by linearization since

$$
2 L\left(c_{1}, c_{2}\right)=L\left(c_{1}+c_{2}, c_{1}+c_{2}\right)-L\left(c_{1}, c_{1}\right)-L\left(c_{2}, c_{2}\right) .
$$

To prove the last equation we apply formula (1.7) with $y=c_{1}, x=c_{2}$ and $z, w \in V$ arbitrary. We get

$$
\begin{aligned}
P(z)\left\{c_{1} c_{2} w\right\} & =-\left\{c_{2} c_{1} P(z) w\right\}+\left\{z w\left\{c_{2} c_{1} z\right\}\right\} \\
& =\left\{c_{2}, c_{1},-P(z) w+\{z w z\}\right\}=\left\{c_{2} c_{1} P(z) w\right\}
\end{aligned}
$$

which shows $\left[P(z), L\left(c_{1}, c_{2}\right)\right]=0$. Now the last equation follows by linearization.

(b) We have for $v \in V$ and $c_{1} \in C(V)$ by applying (1.6) and (a):

$$
\begin{aligned}
L\left(\left\{c_{1} c_{2} c_{3}\right\}, v\right) & =\left[L\left(c_{1}, c_{2}\right), L\left(c_{3}, v\right)\right]+L\left(c_{3},\left\{c_{2} c_{1} v\right\}\right) \\
& =L\left(\left\{c_{2} c_{1} v\right\}, c_{3}\right) \\
& =\left[L\left(c_{2}, c_{1}\right), L\left(v, c_{3}\right)\right]+L\left(v,\left\{c_{1} c_{2} c_{3}\right\}\right) \\
& =L\left(v,\left\{c_{1} c_{2} c_{3}\right\}\right),
\end{aligned}
$$

i.e. $\left\{c_{1} c_{2} c_{3}\right\} \in C(V)$. 
The following lemma will be used in the next section:

LEMMA 3.2. Let $V=S \oplus R$ be a decomposition where $S$ is a semisimple subsystem and $R$ is an ideal of $V$ contained in the center of $V$.

(a) Every derivation of $V$ leaves $S$ invariant.

(b) For $T \in$ End $R$ we define $D_{T} \in$ End $V$ by $D_{T}(s \oplus m)=$ Tm, where $s \in S, m \in R$. Then $D_{T} \in \operatorname{Der} V$ iff

(1) $T \in \operatorname{Der} R$

(2) $T\{s t m\}=\{s t T m\}$ for all $s, t \in S, m \in R$ and

(3) $T\{s m n\}=\{s T m n\}+\{s m T n\}$ for all $s \in S, m, n \in R$.

Proof. (a) We consider $V$ as a bimodule for $S$ by setting $q(x):=P(x)$, $l(x, y)=L(x, y), x, y \in S$, and denote by $\hat{V}$ the split-Null-extension of $S$ by $V$ (see [9] for details). Let $D$ be a derivation of $V$. Then $D: S \rightarrow V$ is a derivation of $S$ into the bimodule $V$. Since $S$ is semisimple it follows from the Remark on page 26 in [9] that there are $s_{1}, \ldots, s_{n} \in S$ and $v_{1}, \ldots, v_{n} \in V$ such that $D s=\sum_{i} \hat{\Delta}\left(s_{i}, v_{i}\right) s$ for $s \in S$ where $\hat{\Delta}\left(s_{i}, v_{i}\right)$ is an inner derivation of $\hat{V}$. Since the product in $\hat{V}=S \oplus V$ satisfies

$$
\begin{aligned}
& \left\{s_{1} \oplus v_{1}, s_{2} \oplus v_{2}, s_{3} \oplus v_{3}\right\} \\
& \quad=\left\{s_{1} s_{2} s_{3}\right\} \oplus\left\{s_{1} s_{2} v_{3}\right\}+\left\{s_{1} v_{2} s_{3}\right\}+\left\{v_{1} s_{2} s_{3}\right\}
\end{aligned}
$$

it follows that

$$
\begin{aligned}
& \hat{\Delta}\left(s_{1} \oplus v_{1}, s_{2} \oplus v_{2}\right)\left(s_{3} \oplus v_{3}\right) \\
& \quad=\Delta\left(s_{1}, s_{2}\right) s_{3} \oplus \Delta\left(s_{1}, s_{2}\right) v_{3}+\Delta\left(s_{1}, v_{2}\right) s_{3}+\Delta\left(v_{1}, s_{2}\right) s_{3} .
\end{aligned}
$$

Hence $\hat{\Delta}\left(s_{l}, v_{i}\right) s=0 \oplus \Delta\left(s_{i}, v_{i}\right) s$. We put $v_{i}=t_{i}+r_{i}$ with $t_{i} \in S$ and $r_{l} \in R$. Then $\Delta\left(s_{i}, v_{i}\right)=\Delta\left(s_{i}, t_{i}\right)$ and thus

$$
D s=\sum_{i} \Delta\left(s_{i}, v_{i}\right) s=\sum_{i} \Delta\left(s_{i}, t_{i}\right) s \in S .
$$

(b) A straightforward computation shows that $D_{T} \in \operatorname{Der} V$ is equivalent to (1), (2), (3) and the following two equations: $T\{s m t\}=\{s T m t\}$, $T\{m s n\}=\{T m s n\}+\{m s T n\}$ for all $s, t \in S, m, n \in R$. But since $R \subset$ $C(V)$ these equations are consequences of (2) and (3).

\section{Jordan triple systems with completely reducible derivation alge-} bras. For the case where the ground field is algebraically closed we will prove the converse of Theorem 2.7 inasmuch as the derivation algebras are concerned. Before stating the theorem we want to recall that we are only dealing with Jordan triple systems which are finite-dimensional over a field of characteristic zero. 
THEOREM 4.1. Let $V$ be a Jordan triple system over an algebraically closed field. Then the following are equivalent:

(a) Der $V$ is completely reducible,

(b) $V$ is a direct sum of two ideals $V^{i}, i=1,2$, where after a possible change of numeration one of the following two cases occurs:

(1) $V^{1}$ is semisimple and $V^{2}$ is trivial,

(2) $V^{1}$ is semisimple and $V^{2}$ is a direct sum of ideals which are isomorphic to an Example 2.3.

Proof. The implication (b) $\Rightarrow$ (a) was already proved in Theorem 2.7. To prove the converse, we proceed in several steps ((4.1) to (4.11)). We put $R=\operatorname{Rad} V$.

$$
\Delta(V, R)=0 \text {, i.e. } R \subset C(V)
$$

Proof. By (1.9) $R$ is invariant under every derivation, hence the formula $[D, \Delta(u, v)]=\Delta(D u, v)+\Delta(u, D v)$ for $D \in \operatorname{Der} V$ implies that $\Delta(V, R)$ is an ideal of Der $V$. Now (1.1) and (1.2) show that $\Delta(V, R)$ is reductive. On the other hand, $\Delta(V, R)$ consists of nilpotent endomorphisms by (1.10) and therefore is by Engel's theorem a nilpotent Lie algebra. It follows that $\Delta(V, R)$ is abelian and, by applying (1.1) once more, every element of $\Delta(V, R)$ is semisimple. Thus $\Delta(V, R)=0$.

$$
\text { If } V=R \text {, then } V \text { is trivial, i.e. }\{\mathrm{VVV}\}=0 \text {. }
$$

Proof. Since $V$ is nilpotent, there exists an $m \in \mathbf{N}$ such that $V_{(2 m-1)} \neq 0$ but $V_{(2 m+1)}=0$. We want to prove $m=1$, so we assume $m \geq 2$. In this case the linear span of $\left\{L(x, y) ; x \in V_{(i)}, y \in V_{(j)}, i+j \geq 2 m-2\right\}$ is abelian by Lemma 3.1.a, contained in Der $V$ because of $\{x y\{u v w\}\} \in$ $V_{(i+j+3)}=0$, and in fact is an ideal of Der $V$ since $D V_{(i)} \subset V_{(i)}$ for every $D \in \operatorname{Der} V$. From (1.1) and (1.2) we derive that $L(x, y)$ for $x \in V_{(i)}$, $y \in V_{(j)}, i+j \geq 2 m-2$, is semisimple and from (1.10) we see that $L(x, y)$ is nilpotent, hence $L(x, y)=0$. Since $V=C(V)$ we have

$$
\{t u\{v w z\}\}=\{t\{u v w\} x\}=\{\{t u v\} w z\}
$$

for every $t, u, v, w, z \in V$, which implies $V_{(2 m-1)}=\left\{V_{(2 m-3)} V V\right\}=0$ because $L\left(V_{(2 m-3)}, V\right)=0$. This is a contradiction and shows $m=1$.

Because of (4.2) we assume in the sequel $V \neq R$. We now come back to the general situation and choose a semisimple subsystem $S$ of $V$ such 
that $V=R \oplus S$, which is possible thanks to (1.13). Then we are in the situation considered in Lemma 3.2.

$$
\text { Der } V=\Delta(V, V) \oplus \operatorname{ex} \operatorname{Der} V \quad \text { (direct sum of ideals) }
$$
where $\Delta(V, V)=\Delta(S, S)=\{D \in \operatorname{Der} V ; \quad D S \subset S$, $D \mid R=0\}$ and ex Der $V:=\{D \in \operatorname{Der} V ; \quad D R \subset R$, $D \mid S=0\}$.

Proof. By Lemma 3.2 every $D \in \operatorname{Der} V$ leaves $S$ and $R$ invariant; hence $D \mid S$ is a derivation which is inner by (1.12), i.e. $D\left|S=\Sigma_{i} \Delta\left(s_{i}, t_{i}\right)\right| S$ for some $s_{i}, t_{i} \in S$. This shows $D=\Sigma_{i} \Delta\left(s_{i}, t_{i}\right)+\left(D-\Sigma_{i} \Delta\left(s_{i}, t_{i}\right)\right)$ with $\Sigma_{i} \Delta\left(s_{i}, t_{l}\right) \in \Delta(V, V)$ and $D-\Sigma_{l} \Delta\left(s_{l}, t_{i}\right) \in \operatorname{ex~Der} V$. Because $\Delta\left(s_{i}, t_{i}\right) R$ $=0$, we have $\Delta(S, S)=\{D \in \operatorname{Der} V ; D S \subset S, D \mid R=0\}$. The equality $\Delta(S, S)=\Delta(V, V)$ is obvious from (4.1). It is also clear that $\Delta(V, V)$ and ex Der $V$ are subalgebras which annihilate each other, thus they are ideals of Der $V$.

In order to extend derivations, Lemma 3.2.b shows that we need to study the operation of the structure algebra of $S$ on $R$. To this end we choose a maximal orthogonal system $\left(e_{1}, \ldots, e_{r}\right)$ of minimal tripotents of $S$. This is possible because the groundfield $k$ is algebraically closed (1.26). We prove next:

$$
L(S, S)\left|S=\sum_{j=1}^{r} k L\left(e_{i}, e_{i}\right)\right| R
$$

Proof. Let $V_{i j}$ resp. $S_{i j}$ be the Peirce spaces of $V$ resp. $S$ relative to $\left(e_{1}, \ldots, e_{r}\right)$. We know $S_{i i}^{+}=k e_{i}$ by (1.20) and $S_{00}=0$ by (1.26). Moreover, for $m \in R$ we have $\left\{e_{i} e_{i} m\right\}=\left\{e_{i} m e_{i}\right\}$. Hence, if $m=m_{2}^{+}+m_{2}^{-}+m_{1}+$ $m_{0}$ is the Peirce decomposition of $m$ relative to $e_{l}$, we see $2 m_{2}^{+}+2 m_{2}^{-}+$ $m_{1}=2 m_{2}^{+}-2 m_{2}^{-}$, which implies $m_{1}=0=m_{2}^{-}$. Therefore

$$
R \subset \bigcap_{i=1}^{r} V_{2}^{+}\left(e_{i}\right) \oplus V_{0}\left(e_{i}\right) .
$$

The definition of the Peirce spaces now implies $R=\oplus_{i=0}^{r} R_{i}$ with $R_{i}=$ $R \cap V_{i t}^{+}$where we put $V_{00}^{+}=V_{00}$. This shows $V_{i i}^{+}=k e_{i} \oplus R_{l}, V_{i i}^{-}=S_{i i}^{-}$ for $1 \leq i \leq r$ and $V_{i j}=S_{i j}$ for $i \neq j$.

We want to investigate $L(S, S) R$ which is a sum of products $\left\{u_{l}, v_{k l} r_{m m}\right\}, u, v \in S, r \in R$. Such a product is zero whenever $k, l \neq m$ and $\{i, j\} \cap\{k, l\}=\varnothing$ set. The remaining cases are products of type $\left\{u_{i j} v_{j m} r_{m m}\right\} \in V_{\mathrm{im}} \cap R=\delta_{\mathrm{im}} R_{m}$. Therefore only $L\left(u_{j m}, v_{j m}\right)$ can operate non-trivially on $R$. Using $R \subset C(V)$ shows that the product $\left\{u_{\jmath m} v_{J m} r_{m m}\right\}$ $\in R_{m}$ also lies in $R_{j}$ which implies $L\left(u_{j m}, v_{j m}\right) R=0$ unless $j=m$. In the 
case $j=m$ we only need to look at a product of the type $\left\{u_{j j} v_{j j} r_{j j}\right\}$. We decompose $u_{j j}=\alpha e_{j}+u_{j j}^{-}, v_{j j}=\beta e_{j}+v_{j j}^{-}$and get

$$
\left\{u_{j j} v_{j j} r_{j j}\right\}=2 \alpha \beta r_{j j}+\alpha\left\{e_{j} v_{j j}^{-} r_{j j}\right\}+\beta\left\{u_{j j}^{-} e_{j} r_{j j}\right\}+\left\{u_{j j}^{-} v_{j j}^{-} r_{j j}\right\} .
$$

But $\left\{e_{j} v_{j j}^{-} r_{j j}\right\}$ and $\left\{u_{j j}^{-} e_{j} r_{j j}\right\} \in V_{j j}^{-} \cap R=0$ which shows $V_{j j}^{-} R_{j}=0$, where the left-hand side is the product in the Jordan algebra $V_{2}\left(e_{j}\right)^{\left(e_{j}\right)}$. Now (1.19) implies $\left\{u_{j j}^{-} v_{j j}^{-} r_{j j}\right\}=-2\left(u_{j j}^{-} v_{j j}^{-}\right) r_{j j}$. But $u_{j j}^{-} v_{j j}^{-} \in k e_{j}$ and therefore $L\left(u_{j j}^{-}, v_{j j}^{-}\right)\left|R \in k L\left(e_{j}, e_{j}\right)\right| R$.

$$
\text { ex Der } V=\operatorname{Der} R_{0} \oplus\left(\oplus_{j=1}^{r} \operatorname{Der}_{2} R_{j}\right) \text { is a direct sum of }
$$
ideals where $D_{j} \in \operatorname{Der}_{2} R_{\mathrm{j}}$ iff $D_{j} \in \operatorname{Der} R_{j}$ and for all $\mathrm{m}, \mathrm{n} \in \mathrm{R}_{\mathrm{j}} \mathrm{D}_{\mathrm{j}}\left\{\mathrm{me}_{\mathrm{j}} \mathrm{n}\right\}=\left\{D_{\mathrm{j}} \mathrm{me}_{\mathrm{j}} \mathrm{n}\right\}+\left\{\mathrm{me}_{\mathrm{j}} \mathrm{D}_{\mathrm{j}} \mathrm{n}\right\}$.

Proof. From (4.3) we know ex $\operatorname{Der} R=\{D \in \operatorname{Der} V ; D R \subset R, D \mid S$ $=0\}$. Applying Lemma 2.3.b and (4.4) shows $D \in \operatorname{ex} \operatorname{Der} R$ iff $D=D_{T}$ where

$$
T \in \operatorname{Der} R,\left[T, L\left(e_{j}, e_{J}\right)\right]=0 \quad \text { for } 1 \leq j \leq r
$$

and

$$
T\{s m n\}=\{s T m n\}+\{s m T n\} \quad \text { for all } s \in S, m, n \in R .
$$

First of all, $\left[T, L\left(e_{j}, e_{j}\right)\right]=0$ for $1 \leq j \leq r$ is equivalent to $T R_{j} \subset R_{j}$ for $0 \leq j \leq r$, i.e. $T=\sum_{j=0}^{r} T_{j}$ where $T_{j}=T \mid R_{j}$. Since $R=\bigoplus_{j=0}^{r} R_{j}$ is a direct sum of ideals we get $T \in \operatorname{Der} R$ iff $T_{j} \in \operatorname{Der} R_{j}$ for $0 \leq j \leq r$. Finally, since $L\left(R_{j}, R_{k}\right)=0$ for $j \neq k$ we have $\{s m n\}=\sum_{j=0}^{r}\left\{s m_{j} n_{j}\right\}=$ $\sum_{j=0}^{r}\left\{m_{j} s_{j j} n_{j}\right\}$. The summand $j=0$ vanishes since $s_{00}=0$, moreover $\left\{m_{j} s_{j j}^{-} n_{j}\right\} \in R_{j} \cap V_{j j}^{-}=0$ and thus $\{s m n\}=\sum_{j=1}^{r}\left\{s_{j j}^{+} m_{j} n_{j}\right\}$. This shows that $T\{s m n\}=\{s T m n\}+\{s m T n\}$ is equivalent to

$$
T_{j}\left\{m e_{j} n\right\}=\left\{T_{j} m e_{j} n\right\}+\left\{m e_{j} T_{j} n\right\} \quad \text { for } 1 \leq j \leq r
$$

and all $m, n \in R_{J}$. The assertion now easily follows.

$$
R_{0} \text { is an ideal of } V \text { with }\left\{R_{0} V V\right\}=0 .
$$

Proof. We have

$$
\left\{V R_{0} V\right\}=\left\{V V R_{0}\right\}=\left\{S S R_{0}\right\}+\left\{S R R_{0}\right\}+\left\{R R R_{0}\right\} .
$$

Here $\left\{S S R_{0}\right\}=0$ by (4.4). Further

$$
\left\{R R_{0} S\right\}=\left\{R_{0} R_{0} S\right\}=\sum_{j=1}^{r}\left\{R_{0} R_{0} S_{0 j}\right\}=\sum_{j=1}^{r}\left\{R_{0} S_{0 j} R_{0}\right\}=0
$$


and

$$
\left\{R R R_{0}\right\}=\left\{R_{0} R_{0} R_{0}\right\}
$$

Hence (4.6) follows if we can show $\left\{R_{0} R_{0} R_{0}\right\}=0$. To this end we note, using (1.2), that Der $R_{0}$ is completely reducible in $R$. Therefore $\left\{R_{0} R_{0} R_{0}\right\}=0$ is a consequence of (4.2).

$$
\{R R V\}=0 \text {. }
$$

Proof. Because of (4.6) and $L\left(R_{j}, R_{k}\right)=0$ for $j \neq k$ it is enough to show $\left\{R_{j} R_{j} V\right\}=0$ for $1 \leq j \leq r$. Here

$$
\left\{R_{j} R_{j} S\right\}=\left\{R_{j} S R_{j}\right\}=\left\{R_{j} S_{j j} R_{j}\right\}=\left\{R_{j} S_{j j}^{+} R_{j}\right\}
$$

since $\left\{R_{j} S_{j j}^{-} R_{j}\right\} \subset R \cap V_{j j}^{-}=0$. Hence $\left\{R_{j} R_{j} S\right\}=0$ iff $\left\{R_{j} e_{j} R_{j}\right\}=0$. But $R_{j} \subset V_{2}^{+}\left(e_{j}\right)$ and thus (1.19) shows that $\left\{R_{j} e_{j} R_{j}\right\}=0$ also implies $\left\{R_{j} R_{j} R_{j}\right\}=0$. We therefore have to prove $\left\{R_{j} e_{j} R_{j}\right\}=0$. We can assume $R_{j} \neq 0$ and for brevity let $N=R_{j}, c=e_{j}$. We define

$$
N^{[0]}=N, \quad N^{[k]}=\left\{N c N^{[k-1]}\right\} \quad \text { for } k \geq 1 .
$$

Since the associative algebra generated by $\{L(a, c) \mid N ; a \in N\}$ is nilpotent ([11] Theorem 14.10), there is an $m \in \mathbf{N}$ such that $N^{[m]}=0$, but $N^{[m-1]} \neq 0$. We assume $m \geq 2$. Then the vector space $l=\{L(a, c) \mid N$; $\left.a \in N^{[m-2]}\right\}$ is well defined. To prove that $l$ consists of derivations of $N$ we first note that the subsystem $k e \oplus N$ coincides with its center, hence, by Lemma 3.1.a and (1.19) we have for $m, n, p \in N$

$$
2\{m n p\}=\{m c\{n c p\}\}=\{\{m c n\} c p\}=\{n c\{m c p\}\} .
$$

This implies for $a \in N^{[m-2]}$ that $\{a c\{m n p\}\} \in R^{[m+1]}=0$ and $\{\{a c m\} n p\}=0$. Thus $L(a, c) \mid N \in \operatorname{Der} N$. Further, by $(*),\{a c\{m c n\}\}=$ $0=\{\{a c m\} c n\}=\{m c\{a c n\}\}$, which shows $L(a, c) \mid N \in \operatorname{Der}_{2} N=$ $\operatorname{Der}_{2} R_{j}$.

Let $D \in \operatorname{Der}_{2} N$. Then $D N^{[k]} \subset N^{[k]}$ and $[D, L(a, c)] \mid N=L(D a, c)$ $\mid N$. Therefore $l$ is an ideal of $\operatorname{Der}_{2} N$ which is abelian, because for $b \in N^{[m-2]}$ and $n \in N$ we have

$$
[L(b, c), L(a, c)] n=L(\{b c a\}, c) n=\{n c\{a c b\}\} \in N^{[m]}=0 .
$$

Since $\operatorname{Der}_{2} N$ is completely reducible in $N$ and $L(a, c) \mid N$ is nilpotent, we conclude from (1.1) that $l=0$, i.e. $N^{[m-1]}=0$ which is a contradiction to our definition of $m$. Thus $m=1$, i.e. $\left\{R_{j} e_{j} R_{j}\right\}=\{N c N\}=0$.

(4.8) direct sum of the semisimple ideal $S$ and the trivial ideal $R_{0}$. 
Proof. We put $R^{+}=\bigoplus_{j=1}^{r} R_{j}$ and compute $\left\{V, V, S \oplus R^{+}\right\}$using (4.7). We get

$$
\{V V S\}+\left\{V V R^{+}\right\}=\{S S S\}+\{R S S\}+\left\{S S R^{+}\right\} \subset S \oplus R^{+}
$$

by (4.4). Similarly, $\left\{V, S \oplus R^{+}, V\right\} \subset S \oplus R^{+}$. Taking into account (4.6) we see that $V=R_{0} \oplus\left(R^{+} \oplus S\right)$ is a direct sum of two ideals. By (4.4) we have $\left(R^{+} \oplus S\right)_{(3)}=R^{+} \oplus S$. Since $R^{+}$is the radical of $R^{+} \oplus S$, we derive from Corollary 2.6, (4.6) and (4.7) that

$$
\operatorname{Der} V=\operatorname{Der}\left(R^{+} \oplus S\right) \oplus \operatorname{Der} R_{0} \oplus \operatorname{Hom}\left(R_{0}, R^{+}\right) \text {. }
$$

Now a comparison with (4.3) and (4.5) shows $\operatorname{Hom}\left(R_{0}, R^{+}\right)=0$. Hence $R^{+}=0$ in case $R_{0} \neq 0$ and (4.8) follows.

In what follows we assume $R_{0}=0$ and show next

$$
\text { If } R_{\mathrm{j}} \neq 0, j \neq 0 \text {, then } V_{j k}=0 \text { for } 0 \leq k \leq r \text { with } k \neq j \text {. }
$$

Proof. First, we look at the case $k=0$ and assume that $V_{0 j}=S_{0 j} \neq 0$. Since $S_{0 j}$ is semisimple by (1.24), it contains a tripotent $f,(1.25)$. By (1.7) we have

$$
\begin{aligned}
\left\{f f e_{j}\right\} & =\left\{f f P\left(e_{j}\right) e_{j}\right\}=-P\left(e_{j}\right)\left\{f f e_{j}\right\}+\left\{e_{j} e_{j}\left\{f f e_{j}\right\}\right\} \\
& =-P\left(e_{j}\right)\left\{f f e_{j}\right\}+2\left\{f f e_{j}\right\}
\end{aligned}
$$

because $\left\{f f e_{j}\right\} \in V_{2}\left(e_{j}\right)$. Thus $P\left(e_{j}\right)\left\{f f e_{j}\right\}=\left\{f f e_{j}\right\} \in S_{2}^{+}\left(e_{j}\right)=k e_{j}$. Since $f \in V_{1}\left(e_{j}\right)$, we know $\left\{f f e_{j}\right\} \neq 0$, and $P(f) e_{j} \in S_{00}=0$ shows $e_{j} \in$ $V_{1}(f) \oplus V_{0}(f)$. Altogether, this gives $\left\{f f e_{j}\right\}=e_{j}$. Now, for $n \in R_{j}$ we get $\{f f n\}=\{f n f\}=0$, but $2 n=\left\{e_{j} e_{j} n\right\}=\left\{e_{j} n e_{j}\right\} \in V_{2}(f)$, a contradiction. Therefore $S_{0 j}=0$.

Let $1 \leq k \leq r$. Then $V_{j k}=V_{j k}^{+} \oplus V_{j k}^{-}$where $V_{j k}^{ \pm}=\left\{x \in V_{j k} ;\left\{e_{j} x e_{k}\right\}\right.$ $= \pm x\}$. It is obviously enough to prove $V_{j k}^{\varepsilon}=0$. We define the Jordan algebras $\mathscr{Q}=\left[V_{2}^{+}\left(e_{j}+\varepsilon e_{k}\right)\right]^{\left(e_{k}+\varepsilon e_{k}\right)}$ and $\mathscr{B}=\left\{S_{2}^{+}\left(e_{j}+\varepsilon e_{k}\right)\right]^{\left(e_{j}+\varepsilon e_{k}\right)}$. Then $\mathscr{B}$ is a subalgebra of the Jordan algebra $Q$ with the same unit element. We assume $V_{j k}^{\varepsilon}=S_{j k}^{\varepsilon} \neq 0$. Then $S_{j k}^{\varepsilon}$ contains an element which is invertible in $\mathscr{B}$ (by [4] VIII Lemma 3.3) and hence also in $\mathcal{Q}$. Thus $P(x) \mid \mathcal{Q}$ is bijective. On the other hand, we derive for $n \in R_{j}$ that $P(x) n=\frac{1}{2}\{x x n\} \in V_{k k} \cap$ $V_{j j}=0$, which forces $n$ to be zero, a contradiction.

(4.10) If $R_{j} \neq 0$, then $V_{2}\left(e_{j}\right)$ is isomorphic to an Example 2.3.

Proof. We know $V_{2}\left(e_{j}\right)=S_{2}\left(e_{j}\right) \oplus R_{j}$. Let $s, t \in S_{2}\left(e_{j}\right)$ and $m$, $n \in R_{j}$. Then, using $\{R R V\}=0$ by (4.7), we derive

$$
P(s \oplus m)(t \oplus n)=P(s) t \oplus P(s) n+\{s t m\} .
$$


Because $S_{2}\left(e_{j}\right)=k e_{j} \oplus S_{2}^{-}\left(e_{j}\right)$ we can apply Lemma 2.1 and (2.3) and conclude that $S_{2}\left(e_{j}\right)$ is the Jordan triple of a non-degenerate symmetric bilinear form $(\cdot, \cdot)$. A comparison with Lemma 2.3 now shows that (4.10) follows if we can prove $P(s) n=(s, s) n$. To this end we decompose $s=\alpha e_{j}+s^{-}$with $\alpha \in k$ and $s^{-} \in S_{2}^{-}\left(e_{j}\right)$. Since $\left\{s^{-} n e_{j}\right\}=0$, we get

$$
P(s) n=\alpha^{2} P\left(e_{j}\right) n+P\left(s^{-}\right) n=\alpha^{2} n+P\left(s^{-}\right) n .
$$

From (1.19) we derive

$$
P\left(s^{-}\right) n=\frac{1}{2}\left\{s^{-} s^{-} n\right\}=-\frac{1}{4}\left\{\left\{s^{-} e_{j} s^{-}\right\} e_{J} n\right\} .
$$

But $\left\{s^{-} e_{j} s^{-}\right\}=2 P\left(s^{-}\right) e_{j}=-2\left(s^{-}, s^{-}\right) e_{j}$ implies

$$
P\left(s^{-}\right) n=\frac{1}{2}\left(s^{-}, s^{-}\right)\left\{e_{j} e_{j} n\right\}=\left(s^{-}, s^{-}\right) n
$$

and $P(s) n=(s, s) n$ follows. Finally, we complete the proof of Theorem 4.1 by showing

$$
\text { Let } A:=\left\{j ; \quad R_{j} \neq 0\right\}, \quad c=\sum_{J \notin A} e_{j}, \quad V^{1}:=V_{2}(c) \oplus
$$

(4.11) $\quad V_{1}(c)$ and $V^{2}=\bigoplus_{j \in A} V_{2}\left(e_{j}\right)$. Then $V=V^{1} \oplus V^{2}$ is a direct sum of ideals where $V^{1}$ is semisimple.

Proof. By (4.9) and the assumption $R_{0}=0$ we get $V=V^{1} \oplus V^{2}$ which is obviously a direct sum of ideals. Since $\operatorname{Rad} V^{1}=V^{1} \cap \operatorname{Rad} V=$ 0 , we see that $V^{1}$ is semisimple.

We come back to the general situation considered in the paper: $V$ is a finite-dimensional Jordan triple system over a field of characteristic zero. In this situation we have

COROLlaRY 4.2. If Der $V$ is completely reducible, then $\operatorname{Rad} V$ is contained in the center of $V$ and $\{V, \operatorname{Rad} V, \operatorname{Rad} V\}=0$.

Proof. Let $K$ be an extension field of $k$. Then $\operatorname{Der}(\mathrm{K} \otimes V)=K \otimes$ Der $V$ by (1.8) and $\operatorname{Der}(K \otimes V)$ is completely reducible in $K \otimes V$ by (1.3). Since $\operatorname{Rad}(K \otimes V)=K \otimes \operatorname{Rad} V$ and $C(K \otimes V)=K \otimes C(V)$ we may assume that $k$ is algebraically closed. But in this case the assertion is just (4.1) and (4.7).

The following theorem is a generalization of a theorem of K.-H. Helwig ([6]) which was quoted in the introduction. 
THEOREM 4.3. For a Jordan triple system $V$ the following statements are equivalent:

(a) Der $V$ is completely reducible and every derivation of $V$ has trace zero,

(b) $V$ is semisimple,

(c) the bilinear form $\left(D_{1}, D_{2}\right) \rightarrow \operatorname{trace}\left(D_{1} D_{2}\right)$ of Der $V$ is non-degenerate, and every derivation of $V$ is inner.

Proof. We put $R=\operatorname{Rad} V$ and choose a semisimple subsystem $S$ of $V$ such that $V=S \oplus R$, which is possible by (1.13). If (a) or (c) holds, then, using Lemma 1.1 in case (c), Der $V$ is completely reducible and we can apply Corollary 4.2 to conclude $R \subset C(V)$ and $\{R R V\}=0$. Let $T=\operatorname{Id}_{R}$. Then $D_{T} \in$ Der $V$ by Lemma 3.2.(b). But obviously $D_{T}$ has trace zero or is inner iff $R=0$, i.e. $V$ is semisimple. Therefore both (a) and (c) imply (b).

If (b) holds, then Der $V$ is completely reducible and the trace form of Der $V$ is non-degenerate by Corollary 1.4. Moreover let $\sigma$ be the trace form of the Jordan triple $V$. Then $\sigma$ is non-degenerate and every derivation of $V$ is skew relative to $\sigma$ and therefore has trace zero. It is also known that every derivation is inner (1.12). Hence (b) implies (a) and (c).

COROLlaRY 4.4. If Der $V$ is semisimple, then $V$ is semisimple too.

5. Real Jordan triple systems with compact automorphism group. We apply Theorem 4.3 to classify real Jordan triple systems with compact automorphism group.

We first recall that a real Jordan triple system is called compact (resp. of non-compact type) if its trace form $\sigma$ is positive-definite (resp. negativedefinite).

THeOREM 5.1. Let $V$ be a real Jordan triple system. Then Aut $V$ is compact iff $V$ is semisimple and for every simple component $V^{i}$ of $V$ one of the following three cases occurs:

(1) $V^{i}$ is compact,

(2) $V^{i}$ is of non-compact type,

(3) $V^{i}$ is isomorphic to the Jordan triple system defined on an Euclidean space $(X,\langle\cdot, \cdot\rangle)$ by

$$
P(x) y=2\langle x, \bar{y}\rangle x-\langle x, x\rangle \bar{y}
$$

where ${ }^{-}$is a non-trivial orthogonal reflection. 
Proof. We assume that Aut $V$ is compact and proceed in several steps: $V$ is semisimple.

Proof. If Aut $V$ is compact, it is well-known that there exists a positive-definite form on $V$ such that Der $V$ consists of skew-adjoint endomorphisms. In particular, Der $V$ is completely reducible and every derivation of $V$ has trace zero. Hence (5.2) is a consequence of Theorem 4.3.

We can assume: $V$ is simple.

Proof. Let $V=V^{1} \oplus \cdots \oplus V^{q}$ be the decomposition of $V$ into its simple components. Then Aut $V^{j}$ is canonically imbedded into Aut $V$ as a closed subgroup, hence Aut $V^{j}$ is compact.

By [15] Satz 3.3 we know that $V$ has a Cartan involution $\alpha$. Let $V=V_{+} \oplus V_{-}$be the corresponding eigenspace decomposition. Then $V_{+}$ is compact and $V_{-}$is of non-compact type. Obviously we may assume $V_{+} \neq 0$ and $V_{-} \neq 0$.

$$
L\left(x_{+}, x_{-}\right)=L\left(x_{-}, x_{+}\right)=P\left(x_{+}, x_{-}\right) \text {for every } x_{ \pm} \in V_{ \pm} .
$$

Proof. We can apply [15] Theorem 2.3.(b) (taking $\lambda=$ trace form) and see that $[D, \alpha]=0$ for every derivation $D$ of $V$. In particular, we get $\alpha \Delta\left(x_{+}, x_{-}\right) \alpha=\Delta\left(x_{+}, x_{-}\right)$. But $\alpha \Delta\left(x_{+}, x_{-}\right) \alpha=-\Delta\left(x_{+}, x_{-}\right)$and hence $\Delta\left(x_{+}, x_{-}\right)=0$, which is equivalent to the first equation of (5.4). To prove the second, let $u=u_{+}+u_{-} \in V$. Then, by applying the first equation (5.4), we get

$$
\begin{aligned}
\left\{x_{+} x_{-} u\right\} & =\left\{x_{+} x_{-} u_{+}\right\}+\left\{x_{+} x_{-} u_{-}\right\}=\left\{x_{+} u_{+} x_{-}\right\}+\left\{x_{-} x_{+} u_{-}\right\} \\
& =\left\{x_{+} u_{+} x_{-}\right\}+\left\{x_{-} u_{-} x_{+}\right\}=\left\{x_{+} u x_{-}\right\}
\end{aligned}
$$

i.e. $L\left(x_{+}, x_{-}\right)=P\left(x_{+}, x_{-}\right)$.

We recall from [12] $\$ 1.1$ that every element $x \in V_{+}$has a minimal decomposition, i.e. $x=\sum_{j=1}^{n} \lambda_{j} e_{j}$ where $\lambda_{j} \in \mathbf{R}$ and $\left(e_{1}, \ldots, e_{n}\right)$ is an orthogonal system of minimal tripotents. In particular, $V_{+}$has many tripotents.

If $c$ is a tripotent in $V_{+}$, then $V_{-}=\left(V_{-} \cap V_{2}^{+}(c)\right) \oplus$ $\left(V_{-} \cap V_{0}(c)\right)$.

Proof. Let $x_{-} \in V_{-}$have the Peirce decomposition $x_{-}=y_{2}^{+}+y_{2}^{-}+$ $y_{1}+y_{0}$ with $y_{2}^{ \pm} \in V_{2}^{ \pm}(c)$ and $y_{t} \in V_{i}(c), i=1,0$. Then $\left\{c x_{-} c\right\}=\left\{x_{-} c c\right\}$ 
by (5.4). But the left hand side equals $2 y_{2}^{+}-2 y_{2}^{-}$, whereas the right hand side is $2 y_{2}^{+}+2 y_{2}^{-}+y_{1}$. Hence $y_{2}^{-}=0=y_{1}$. Since $L(c, c) V_{-} \subset V_{-}$and $P(c) V_{-} \subset V_{-}$, we get the claimed splitting of $V_{-}$.

$$
\begin{aligned}
& V=V_{2}(c) \text { where } c \text { is a tripotent of } V_{+} \text {which is minimal } \\
& \text { in } V_{+} \text {. }
\end{aligned}
$$

Proof. First, we assume: For every minimal tripotent $c$ of $V_{+}$the Peirce space $V_{1}(c)$ is non-zero.

Let $x \in V_{-} \cap V_{2}(c), c$ a minimal tripotent of $V_{+}$. Then $L(c, x) V_{1}(c)$ $=L(x, c) V_{1}(c)=P(x, c) V_{1}(c)=0$. Hence

$$
V_{-} \cap V_{2}(c) \subset I_{2}(c)=\left\{y \in V_{2}(c) ; L(y, c) V_{1}(c)=L(c, y) V_{1}(c)=0\right\} \text {. }
$$

But $I_{2}(c)$ is an ideal of $V_{2}(c)$ ([16] Corollary 1.8) and $V_{2}(c)$ is simple by [13]. If $I_{2}(c)=V_{2}(c)$, then $c \in I_{2}(c)$ implies $V_{1}(c)=0$. Therefore, by our assumption, $V_{-} \cap V_{2}(c) \subset I_{2}(c)=0$ and, by (5.5), $V_{-} \subset V_{0}(c)$. From the Peirce multiplication rules now follows $L\left(c, V_{-}\right)=0$. Since this holds for every minimal tripotent of $V_{+}$and since every element of $V_{+}$has a minimal decomposition as mentioned above, we conclude $L\left(V_{+}, V_{-}\right)=0$ $=P\left(V_{+}, V_{-}\right)$. This implies that $V=V_{+} \oplus V_{-}$is a direct sum of two non-trivial ideals, a contradiction to (5.3).

Therefore, there exists a minimal tripotent $c$ of $V_{+}$such that $V_{1}(c)=0$. Since $V$ is simple, this forces $V_{0}(c)$ to vanish and (5.6) follows.

We denote by $X_{+}$the underlying vector space of the Jordan triple system $V_{+}$. Then

There exists a scalar product $\langle\cdot, \cdot\rangle$ on $X_{+}$such that $V_{+}$ is the Jordan triple system associated to $\langle\cdot, \cdot\rangle$. Moreover, $\left\{x_{+} y_{+} y_{+} z_{-}\right\}=2\left\langle x_{+}, y_{+}\right\rangle z_{-}$.

Proof. By (5.6) we know $V_{+}=\left(V_{+}\right)_{2}(c)$ where $c$ is a minimal tripotent of $V_{+}$. Therefore $\left(V_{+}\right)_{2}^{+}(c)=\mathbf{R} c$ and Lemma 2.1 says that $V_{+}$ is the Jordan triple associated to a bilinear form $\langle\cdot, \cdot\rangle$ on $X_{+}$. It is easy to compute that the trace form $\sigma$ of $V_{+}$is

$$
\sigma(x, y)=\frac{1}{2} \operatorname{trace}(L(x, y)+L(y, x))=2\langle x, y\rangle \operatorname{dim} X_{+}
$$

which implies that $\langle\cdot, \cdot\rangle$ is positive-definite.

Let $d \in V_{+}$and assume $\langle d, d\rangle=1$. Then by (2.4) $d$ is a tripotent of $V_{+}=\left(V_{+}\right)_{2}(d)$. In particular, $c \in V_{2}(d)$ and $L(c, c)\left(V_{-} \cap V_{0}(d)\right)=0$. But $V_{-} \subset V_{2}(c)$ and therefore $L(c, c) \mid\left(V_{-} \cap V_{0}(d)\right)=2$ Id which shows $V_{-} \cap V_{0}(d)=0$ and, by (5.5), $V_{-} \subset V_{2}^{+}(d)$. Hence, for $x_{+} \in V_{+}$arbitrary, $\left\{x_{+} x_{+} z_{-}\right\}=2\left\langle x_{+}, x_{+}\right\rangle z_{-}$. Since $\left\{x_{+} y_{+} z_{-}\right\}=\left\{y_{+} x_{+} z_{-}\right\}$, we get by linearization $\left\{x_{+} y_{+} z_{-}\right\}=2\left\langle x_{+}, y_{+}\right\rangle z_{-}$. 
We denote by $X_{-}$the underlying vector space of the Jordan triple $V_{-}$. Then

There exists a scalar product $\langle\cdot, \cdot\rangle$ on $X_{-}$such that $V_{-}$ (5.8) is the Jordan triple associated to $-\langle\cdot, \cdot\rangle$. Moreover, $\left\{x_{-} y_{-} z_{+}\right\}=-2\left\langle x_{-}, y_{-}\right\rangle z_{+}$.

Proof. Let $\{\cdots\}$ denote the Jordan triple product of $V$ and $V^{-}$the Jordan triple system with triple product $-\{\cdots\}$. Then $V^{-}$is simple, Aut $V^{-}=$Aut $V$ is compact and $-\alpha$ is a Cartan involution of $V$, hence . $\left(V^{-}\right)_{+}=V_{-},\left(V^{-}\right)_{-}=V_{+}$. We therefore can apply (5.7) to $V_{-}$and get (5.8).

Let $X$ be the underlying vector space of $V$. Then $X$ has a scalar product $\langle\cdot, \cdot\rangle$, given by $\left\langle x_{+}+x_{-}, y_{+}+y_{-}\right\rangle=\left\langle x_{+}, y_{+}\right\rangle+\left\langle x_{-}, y_{-}\right\rangle$. We define $\bar{x}=\overline{x_{+}+x_{-}}=x_{+}-x_{-}$. Then ${ }^{-}$is a nontrivial orthogonal reflection. Moreover, we have for $x, y, z \in V$

$$
\{x y z\}=2[\langle x, \bar{y}\rangle z+\langle z, \bar{y}\rangle x-\langle x, z\rangle \bar{y}] \text {. }
$$

Proof. From (5.7) resp. (5.8) and (2.1) we derive

$$
\left\{x_{\varepsilon} y_{\varepsilon} z_{\varepsilon}\right\}=2 \varepsilon\left[\left\langle x_{\varepsilon}, y_{\varepsilon}\right\rangle z_{\varepsilon}+\left\langle z_{\varepsilon}, y_{\varepsilon}\right\rangle x_{\varepsilon}-\left\langle x_{\varepsilon}, z_{\varepsilon}\right\rangle y_{\varepsilon}\right] \text {. }
$$

Moreover, by (5.4), we know $\left\{x_{+} y_{+} z_{-}\right\}=\left\{x_{+} z_{-} y_{+}\right\}=\left\{z_{-} x_{+} y_{+}\right\}$ and $\left\{x_{-} y_{-} z_{+}\right\}=\left\{x_{-} z_{+} y_{-}\right\}=\left\{z_{+} x_{-} y_{-}\right\}$. Hence, by (5.7) resp. (5.8), we get for $\varepsilon= \pm$ :

$$
\begin{aligned}
& \left\{x_{\varepsilon} y_{\varepsilon} z_{-\varepsilon}\right\}=2 \varepsilon\left\langle x_{\varepsilon}, y_{\varepsilon}\right\rangle z_{-\varepsilon}, \\
& \left\{x_{\varepsilon} y_{-\varepsilon} z_{\varepsilon}\right\}=2 \varepsilon\left\langle x_{\varepsilon}, z_{\varepsilon}\right\rangle y_{-\varepsilon} \text { and } \\
& \left\{x_{-\varepsilon} y_{\varepsilon} z_{\varepsilon}\right\}=2 \varepsilon\left\langle y_{\varepsilon}, z_{\varepsilon}\right\rangle x_{-\varepsilon} .
\end{aligned}
$$

It is now a straightforward verification, that in all cases the triple product $\left\{x_{\varepsilon} y_{\mu} z_{\delta}\right\}, \varepsilon, \mu, \delta= \pm$, can be expressed by the right-hand side of (5.9). This proves (5.9).

Since $P(x) y=\frac{1}{2}\{x y x\}=2\langle x, \bar{y}\rangle x-\langle x, x\rangle \bar{y}$ by (5.9) we have finally proved one direction of Theorem 5.1. We consider now the other direction: Let $V=V^{1} \oplus \cdots \oplus V^{q}$ be the decomposition of $V$ into its simple ideals and $G=\left(\right.$ Aut $\left.V^{1}\right) \times \cdots \times\left(\right.$ Aut $\left.V^{q}\right)$. Then Aut $V / G$ is finite, since it is represented by automorphisms which map isomorphic ideals onto each other. It is therefore enough to show that Aut $V^{j}$ is compact for $1 \leq j \leq q$. This is clear if $V^{j}$ is compact or of non-compact type since 
every automorphism is orthogonal relative to the trace form. Hence it remains to prove that Aut $V$ is compact for a Jordan triple $V$ given by (5.9).

Let $\varphi \in$ Aut $V$. Then $\varphi$ is orthogonal relative to the trace form $\sigma$ of $V$ which is $\sigma(x, y)=2\langle x, \bar{y}\rangle \operatorname{dim} V$. Hence $\langle x, \bar{y}\rangle=\langle\varphi x, \overline{\varphi y}\rangle$ for $x, y \in V$. Moreover

$$
\begin{aligned}
& 2\langle x, \bar{y}\rangle \varphi x-\langle x, x\rangle \varphi(\bar{y})=\varphi(P(x) y) \\
& \quad=P(\varphi x) \varphi y=2\langle\varphi x, \overline{\varphi y}\rangle \varphi x-\langle\varphi x, \varphi x\rangle \overline{\varphi y}
\end{aligned}
$$

implies $\langle x, x\rangle \varphi(\bar{y})=\langle\varphi x, \varphi x\rangle \overline{\varphi y}$ for all $x, y \in V$, i.e. $\varphi(\bar{y})=\alpha \overline{\varphi y}$ with $\alpha \in \mathbf{R}, \alpha>0$. Taking the determinant shows $\alpha=1$. Thus $\varphi$ is also orthogonal relative to $\langle\cdot, \cdot\rangle$. Conversely, it is obvious that a map with $\varphi(\bar{y})=\overline{\varphi(y)}$ and $\langle\varphi x, \varphi y\rangle=\langle x, y\rangle$ for every $x, y \in V$ is an automorphism of $V$, i.e. Aut $V=\{\varphi \in 0(V,\langle\cdot, \cdot\rangle) ; \varphi(\bar{y})=\overline{\varphi(y)}$ for all $y \in V\}$ which clearly is a compact group.

6. Jordan triple systems with completely reducible structure algebras. As a further application of Theorem 4.1 we will classify Jordan triple systems with completely reducible structure algebras, even in the case where the ground field is not algebraically closed. The link to Theorem 4.1 is provided by the following

LEMMA 6.1. If $\mathcal{T}(V)$ is completely reducible, then Der $V$ is completely reducible..

Proof. Since $\mathcal{T}(V)$ is completely reducible there is a decomposition $V=W^{1} \oplus \cdots \oplus W^{q}$ where the $W^{i}$ are $\mathcal{T}(V)$-irreducible subspaces. This yields a decomposition

$$
(V, V)=\left(W^{1}, 0\right) \oplus \cdots \oplus\left(W^{q}, 0\right) \oplus\left(0, W^{1}\right) \oplus \cdots \oplus\left(0, W^{q}\right)
$$

into $\operatorname{Der}(V, V)$-irreducible subspaces. Hence $\operatorname{Der}(V, V)$ is completely reducible.

The map $\theta: \operatorname{Der}(V, V) \rightarrow \operatorname{Der}(V, V):(A, B) \rightarrow(B, A)$ is an involutive automorphism of $\operatorname{Der}(V, V)$ inducing a decomposition

$$
\operatorname{Der}(V, V)=\mathfrak{K} \oplus \Re
$$

where $\mathcal{K}=\{(A, A) \in \operatorname{Der}(V, V)\}$ and $\mathscr{K}=\{(A,-A) \in \operatorname{Der}(V, V)\}$. Since $\operatorname{Der}(V, V)$ is completely reducible, every nilpotent element can be imbedded in a three-dimensional split simple subalgebra of $\operatorname{Der}(V, V)([8]$ III Theorem 17.1). Because [ $\mathscr{K}, \mathscr{K}] \subset \mathscr{K}$ and $[\mathscr{K}, \mathscr{K}] \subset \mathscr{K}$ the same 
property holds for $\mathscr{K}$ by [8] III Lemma 8 . Moreover $\mathscr{K}$ is almost algebraic and thus the center of $\mathscr{K}$ is almost algebraic, too. Thus [8] III Theorem 17.2 implies that $\mathscr{K}$ is completely reducible.

Now $\mathcal{K} \rightarrow \operatorname{Der} V:(A, A) \rightarrow A$ is an isomorphism, in particular Der $V$ is reductive. If $D$ is in the center of $\operatorname{Der} V,(D, D)$ is in the center of $\mathscr{K}$ and thus semisimple, which shows that $D$ is semisimple. By (1.1) Der $V$ is completely reducible.

THEOREM 6.2. For a Jordan triple system $V$ the following are equivalent:

(a) $\mathcal{T}(V)$ is completely reducible,

(b) $V$ is the direct sum of a semisimple and a trivial ideal.

Proof. Since $(b) \Rightarrow$ (a) was already proved in Theorem 2.7 it remains to show (a) $\Rightarrow(\mathrm{b})$.

We first consider the case where the ground field $k$ is algebraically closed. Since Der $V$ is completely reducible we can apply Theorem 4.1 which in conjunction with Theorem 2.7(b) shows (b), i.e.

$$
V=\{V V V\} \oplus \operatorname{Rad} V
$$

where $\{V V V\}$ is a semisimple ideal and $\operatorname{Rad} V$ is a trivial ideal.

In general, let $\bar{k}$ be an algebraic closure of $k$. Since $\bar{k} \otimes\{V V V\}=$ $\{\bar{k} \otimes V, \bar{k} \otimes V, \bar{k} \otimes V\}$ we see that $\{V V V\}$ is a semisimple deal, similarly $\operatorname{Rad} V$ is a trivial ideal. Because $\bar{k} \otimes V=(\bar{k} \otimes\{V V V\}) \oplus(\bar{k} \otimes \operatorname{Rad} V)$ we get $V=\{V V V\} \oplus \operatorname{Rad} V$.

REMARK 6.3. If $V=I^{1} \oplus I^{2}$ is a direct sum of ideals where $I^{1}$ is semisimple and $I^{2}$ is trivial, then $I^{1}=\{V V V\}$ and $I^{2}=\operatorname{Rad} V$, i.e. the decomposition in Theorem 6.2(b) is unique.

It seems to be worthwhile to mention the following

COROLlARY 6.4. Let $q$ be a unital Jordan algebra. Then $q$ is semisimple iff the structure algebra of $q$ is completely reducible.

\section{REFERENCES}

[1] N. Bourbaki, Algèbre, Chap. 8 Hermann, Paris 1958.

[2] _ Groupes et algèbres de Lie, Chap. 1, Hermann, Paris 1971.

[3] _ Groupes et algèbres de Lie, Chap. 7 et 8. Hermann, Paris 1975.

[4] H. Braun and M. Koecher, Jordan-Algebren, Springer-Verlag, Berlin-Heidelberg-New York 1966. 
[5] C. Chevalley, Théorie des groupes de Lie, tome II. Hermann, Paris 1951.

[6] K.-H. Helwig, Halbeinfache reelle Jordan-Algebren, Habilitationsschrift, München 1968.

[7] C. Hochschild, Semi-simple algebras and generalized derivations, Amer. J. Math., 64 (1942), 677-694.

[8] N. Jacobson, Lie Algebras, Interscience Publishers, New York-London-Sydney 1962.

[9] O. Kühn and A. Rosendahl, Wedderburnzerlegung für Jordan-Paare, Manuscripta Math., 24 (1978), 403-435.

[10] O. Loos, Lectures on Jordan triples, Lecture notes, The University of British Columbia, Vancouver 1971.

[11] Jordan Pairs, Lecture notes in mathematics 460, Springer-Verlag, BerlinHeidelberg-New York 1975.

[12] _ Bounded symmetric domains and Jordan pairs, Lecture notes, University of California at Irvine 1977.

[13] K. McCrimmon, Peirce ideals in Jordan triple systems, Pacific J. Math., 83 (1979), 415-439.

[14] K. Meyberg, Lectures on algebras and triple systems, Lecture notes, The University of Virginia, Charlottesville 1972.

[15] E. Neher, Cartan-Involutionen von halbeinfachen reellen Jordan-Tripelsystemen, Math. Z., 169 (1979), 271-292.

[16] _ Involutive gradings of Jordan structures, Comm. Algebra, 9 (1981), 575-599.

[17] H. P. Petersson, Conjugacy of idempotents in Jordan pairs, Comm. Algebra, 6 (1978), 673-715.

[18] R. D. Schafer, A theorem on the derivations of Jordan algebras, Proc. Amer. Math. Soc., 2 (1951), 290-294.

Received April 6, 1982 and in revised form January 27, 1983. Partially supported by Deutsche Forschungsgemeinschaft.

MATH. INSTITUT DER UNIVERSITÄT

EINSTEINSTR. 64/IV

4400 MUNSTER

WEST-GERMANY

Current address: Department of Mathematics

University of Ottawa

Ottawa, Ontario K1N 9B4

Canada 


\section{PACIFIC JOURNAL OF MATHEMATICS \\ EDITORS}

Donald BABBITT (Managing Editor)

University of California

Los Angeles, CA 90024

Hugo Rossi

University of Utah

Salt Lake City, UT 84112

C. C. Moore and Arthur Ogus

University of California

Berkeley, CA 94720
J. DugunduI

Department of Mathematics

University of Southern California

Los Angeles, CA 90089-1113

R. FinN and H. SAMELSON

Stanford University

Stanford, CA 94305

ASSOCIATE EDITORS

R. ARENS

E. F. BECKENBACH

B. H. NeUMANN

F. WOLF

K. YosHIDA (1906-1982)

\section{SUPPORTING INSTITUTIONS}

UNIVERSITY OF ARIZONA

UNIVERSITY OF BRITISH COLUMBIA

CALIFORNIA INSTITUTE OF TECHNOLOGY

UNIVERSITY OF CALIFORNIA

MONTANA STATE UNIVERSITY

UNIVERSITY OF NEVADA, RENO

NEW MEXICO STATE UNIVERSITY

OREGON STATE UNIVERSITY
UNIVERSITY OF OREGON

UNIVERSITY OF SOUTHERN CALIFORNIA

STANFORD UNIVERSITY

UNIVERSITY OF HAWAII

UNIVERSITY OF TOKYO

UNIVERSITY OF UTAH

WASHINGTON STATE UNIVERSITY

UNIVERSITY OF WASHINGTON 


\section{Pacific Journal of Mathematics}

\section{Vol. 113, No. $1 \quad$ March, 1984}

Flavio E. A. da Silveira, Rational homotopy theory of fibrations $\ldots \ldots \ldots \ldots 1$

Donald M. Davis, Desuspensions of stunted projective spaces ............ 35

Lou van den Dries, Exponential rings, exponential polynomials and

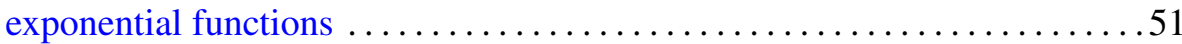

Fred Galvin and Samuel David Shore, Completeness in semimetric

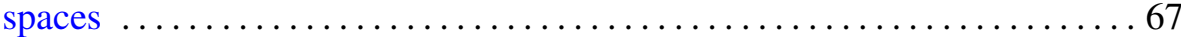

Fereidoun Ghahramani, Compact elements of weighted group algebras . . . 777

Munehiko Itōo, The closed image of a hereditary $M_{1}$-space is $M_{1} \ldots \ldots \ldots 85$

Elvira Laura Livorni, Classification of algebraic surfaces with sectional genus less than or equal to six. I. Rational surfaces .............. 93

H. Alan MacLean, Riesz sets and a theorem of Bochner ............ 115

E. Neher, Jordan triple systems with completely reducible derivation or

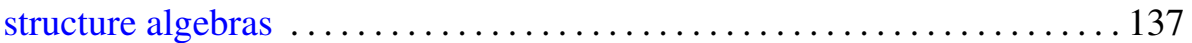

Joe Repka, Shalika's germs for $p$-adic GL( $n)$. I. The leading term $\ldots \ldots \ldots 165$

Joe Repka, Shalika's germs for $p$-adic GL( $n)$. II. The subregular term $\ldots \ldots 173$

Rae Michael Andrew Shortt, Borel density, the marginal problem and isomorphism types of analytic sets $\ldots \ldots \ldots \ldots \ldots \ldots \ldots \ldots \ldots \ldots \ldots \ldots \ldots$

Baruch Solel, The multiplicity functions of invariant subspaces for nonselfadjoint crossed products $\ldots \ldots \ldots \ldots \ldots \ldots \ldots \ldots \ldots \ldots \ldots \ldots . \ldots \ldots$

Su-win Yang, Self-intersection number of immersions and enumeration of nonstable vector bundles

W. M. Zajączkowski, Local solvability of nonstationary leakage problem for ideal incompressible fluid. II 